\section{Bortezomib with standard chemotherapy for children with acute myeloid leukemia does not improve treatment outcomes: a report from the Children's Oncology Group}

\author{
Richard Aplenc, ${ }^{1}$ Soheil Meshinchi, ${ }^{2 *}$ Lillian Sung, ${ }^{{ }^{*}}$ Todd Alonzo, ${ }^{4}$ John Choi, ${ }^{5}$ \\ Brian Fisher, ${ }^{6}$ Robert Gerbing, ${ }^{7}$ Betsy Hirsch, ${ }^{8}$ Terzah Horton, ${ }^{9}$ \\ Samir Kahwash, ${ }^{10}$ John Levine,${ }^{11}$ Michael Loken, ${ }^{12}$ Lisa Brodersen, ${ }^{12}$ \\ Jessica Pollard, ${ }^{13}$ Susana Raimondi, ${ }^{5}$ Edward Anders Kolb ${ }^{14}$ and Alan Gamis ${ }^{15}$
}

${ }^{*} R A$ and $S M$ contributed equally to this work.

${ }^{1}$ The Children's Hospital of Philadelphia, Division of Oncology, Philadelphia, PA, USA; ${ }^{2}$ Fred Hutchinson Cancer Research Center, Seattle, WA, USA; ${ }^{3}$ The Hospital for Sick Children, Toronto, ON, Canada; ${ }^{4}$ University of Southern California, Los Angeles, CA, USA; ${ }^{5}$ St. Jude Children's Research Hospital, Memphis, TN, USA; 'The Children's Hospital of Philadelphia, Division of Infectious Disease, Philadelphia, PA, USA; ${ }^{7}$ Children's Oncology Group, Monrovia, CA, USA; ${ }^{2}$ University of Minnesota, Minneapolis, MN, USA; ${ }^{9}$ Texas Children's Hospital, Houston, TX, USA; ${ }^{10}$ Nationwide Children's Hospital, Columbus, $\mathrm{OH}$, USA; ${ }^{11}$ Mount Sinai Medical Center, New York, NY, USA; ${ }^{12}$ Hemaologics Inc., Seattle, WA, USA; ${ }^{13}$ Dana Farber Cancer Center, Boston, MA, USA; ${ }^{14}$ Alfred I. duPont Hospital for Children, Wilmington, DE, USA and ${ }^{15}$ Children's Mercy Hospital and Clinics, Kansas City, MO, USA

\section{ABSTRACT}

$\mathrm{N}$ ew therapeutic strategies are needed for pediatric acute myeloid leukemia (AML) to reduce disease recurrence and treatment-related morbidity. The Children's Oncology Group Phase III AAML1031 trial tested whether the addition of bortezomib to standard chemotherapy improves survival in pediatric patients with newly diagnosed AML. AAML1031 randomized patients younger than 30 years of age with de novo AML to standard treatment with or without bortezomib. All patients received the identical chemotherapy backbone with either four intensive chemotherapy courses or three courses followed by allogeneic hematopoietic stem cell transplantation for high-risk patients. For those randomized to the intervention arm, bortezomib $1.3 \mathrm{mg} / \mathrm{m}^{2}$ was given on days 1,4 and 8 of each chemotherapy course. For those randomized to the control arm, bortezomib was not administered. In total, 1,097 patients were randomized to standard chemotherapy $(\mathrm{n}=542)$ or standard chemotherapy with bortezomib $(\mathrm{n}=555)$. There was no difference in remission induction rate between the bortezomib and control treatment arms ( $89 \%$ vs. $91 \%, P=0.531)$. Bortezomib failed to improve 3-year event-free survival $(44.8 \pm 4.5 \%$ vs. $47.0 \pm 4.5 \%$, $P=0.236)$ or overall survival (63.6 \pm 4.5 vs. $67.2 \pm 4.3, P=0.356)$ compared with the control arm. However, bortezomib was associated with significantly more peripheral neuropathy $(P=0.006)$ and intensive care unit admissions $(P=0.025)$ during the first course. The addition of bortezomib to standard chemotherapy increased toxicity but did not improve survival. These data do not support the addition of bortezomib to standard chemotherapy in children with de novo AML. (Trial registered at clinicaltrials.govNCT01371981; https://www.cancer.gov/clinicaltrials/ NCT01371981).

\section{Introduction}

Pediatric acute myeloid leukemia (AML) is the second most common pediatric leukemia and requires intensive therapy for cure. ${ }^{1,2}$ Despite the intensity of AML chemotherapy, which includes a very high cumulative lifetime anthracycline exposure in patients treated with chemotherapy alone or allogeneic donor stem cell transplantation (SCT) in first remission, approximately $50 \%$ of patients will experi-
Haematologica 2020

Volume 105(7):1879-1886

\section{Correspondence:}

RICHARD APLENC

aplenc@email.chop.edu

Received: March 7, 2019.

Accepted: February 5, 2020.

Pre-published: February 6, 2020.

doi:10.3324/haematol.2019.220962

Check the online version for the most updated information on this article, online supplements, and information on authorship \& disclosures: www.haematologica.org/content/105/7/1879

\section{(C)2020 Ferrata Storti Foundation}

Material published in Haematologica is covered by copyright. All rights are reserved to the Ferrata Storti Foundation. Use of published material is allowed under the following terms and conditions:

https://creativecommons.org/licenses/by-nc/4.0/legalcode. Copies of published material are allowed for personal or internal use. Sharing published material for non-commercial purposes is subject to the following conditions: https://creativecommons. org/licenses/by-nc/4.0/legalcode sect. 3. Reproducing and sharing published material for commercial purposes is not allowed without permission in writing from the publisher. 
ence disease recurrence. ${ }^{3,4}$ Moreover, treatment-related mortality limits the ability to further intensify therapy. ${ }^{5}$ Thus, new therapies are needed to improve the outcomes of children with AML.

The development and evaluation of targeted therapies for children with AML is the highest clinical research priority for the Myeloid Committee in the Children's Oncology Group (COG). ${ }^{6}$ After successfully demonstrating an improvement in event-free survival (EFS) in children treated with gemtuzumab, ${ }^{3,4}$ COG sought to evaluate the efficacy of bortezomib, a first-generation proteasome inhibitor approved for multiple myeloma and nonHodgkin lymphoma. Bortezomib was selected based on preliminary data demonstrating that AML blasts have increased proteasomes and are more sensitive to proteasome inhibitor-mediated apoptosis, ${ }^{7}$ AML stem cells have increased NF- $\mathrm{\kappa B}$ that is selectively targeted with proteasome inhibitors, ${ }^{8-11}$ preclinical data from the pediatric preclinical testing program showing activity against leukemia cell lines, ${ }^{12,13}$ and studies in adults with AML demonstrating clinical benefit. ${ }^{14-16}$ At the time of the opening of the AAML1031 study, a COG pediatric phase I single agent bortezomib trial had determined the single agent maximum tolerated dose ${ }^{17}$ and a phase II trial (AAML07P1), combining bortezomib with AML chemotherapy for patients with relapsed AML, was nearing completion. ${ }^{18}$

Since the available safety and efficacy data for combining bortezomib with standard AML chemotherapy was limited, COG, in collaboration with the Cancer Therapy Evaluation Program (CTEP), designed AAML1031 as a definitive efficacy phase III trial with an interim toxicity analysis to ensure that combining bortezomib with standard AML chemotherapy was safe. The primary objective of AAML1031 was to definitively assess the impact of bortezomib in combination with standard AML chemotherapy on EFS for children with newly diagnosed AML without high allelic ratio (HAR) FLT3 ITD. A second objective was to evaluate the impact of bortezomib on overall survival (OS). Based on the available preliminary data at the time of study initiation, bortezomib was hypothesized to improve both EFS and OS. Multiple secondary objectives included an expanded safety assessment, multiple biology correlative studies, and secondary clinical data analyses.

\section{Methods}

The AAML1031 study was an open-label multi-center randomized trial including patients aged 0 to 29.5 years with previously untreated primary AML. Exclusion criteria were: prior chemotherapy, acute promyelocytic leukemia [t(15;17)], juvenile myelomonocytic leukemia, bone marrow failure syndromes, or secondary AML. The National Cancer Institute's central institutional review board (IRB) and IRB at each enrolling center approved the study; patients and families provided informed consent or assent as appropriate. The trial was conducted in accordance with the Declaration of Helsinki. The trial was registered at clinicaltrials.gov identifier: NCT01371981.

Patients were randomly assigned at enrollment to either standard AML treatment or standard treatment with bortezomib. Randomization was conducted in blocks of four. Bortezomib was administered at a dose of $1.3 \mathrm{mg} / \mathrm{m}^{2}$ once on days 1,4 , and 8 of each chemotherapy course.

Patients with high allelic ratio FLT3 ITD were offered enroll- ment on a phase I sorafenib treatment arm if that arm was open. Patients with HAR FLT3 ITD who declined enrollment in the sorafenib arm, or who enrolled while the arm was suspended, continued to receive treatment according to their initial randomization. These patients were included in safety analyses but were excluded from all efficacy analyses.

Patients were classified as low- or high-risk after Induction I. Low-risk patients received four courses of chemotherapy and high-risk patients received three courses of chemotherapy followed by allogeneic SCT. High-risk patients without an appropriate donor received four courses of chemotherapy.

The primary end point was EFS from study entry. EFS was defined as the time from study entry until death, refractory disease, or relapse of any type, whichever occurred first. The secondary end points were OS, remission rates, relapse risk, post induction disease-free survival (DFS), and treatment-related mortality (TRM). OS was defined as time from study entry until death. Relapse risk was defined as the time from the end of Induction II for patients in complete remission (CR) to relapse, where deaths without a relapse were considered competing events. DFS was defined as the time from end of Induction II for patients in CR until relapse or death. Refractory disease was defined as the persistence of central nervous system (CNS) disease after Induction I, or the presence of morphologic bone marrow blasts $\geq 5 \%$ or any extramedullary disease at the end of Induction II. Patients with refractory disease were removed from protocol therapy. TRM was defined as the time from either study entry, or from end of Induction II for patients in CR, to deaths without a relapse, with relapses considered as competing events. Patients without an event were censored at their date of last known contact. However, for TRM analyses, patients were censored 30 days post end of therapy or 200 days post SCT.

\section{Statistical analysis}

The study was designed with 1 -sided testing and $2.5 \%$ type I error rate and $80 \%$ power to detect a $9 \%$ difference in EFS plateaus $(52 \%$ vs. $61 \%$, hazard ratio $=0.78)$ between patients without HAR FLT3 ITD randomized to standard therapy versus bortezomib/standard combination therapy. All $P$-values are twosided. Please see the Online Supplementary Appendix for additional details of the methods used.

\section{Results}

Between February 2011 and January 2016, 1,231 patients were enrolled on the AAML1031 study; patients were aged 0 to 29.5 years and had previously untreated primary AML at 184 institutions. Data for this analysis were frozen at December 31, 2017, with a median followup period of 3.0 years (range, 0-6.0 years) for patients alive at last contact. A total of 132 patients were excluded: 32 patients not meeting eligibility criteria, 102 patients with HAR FLT3 ITD who either enrolled $(n=60)$ or did not enroll $(n=42)$ on the phase I sorafenib treatment arm that concluded enrollment on July 31, 2017; this left 1,097 patients eligible for analysis. Figure 1 illustrates the reasons for exclusion and shows that 555 participants were randomized to the bortezomib arm and 542 to the control arm.

Accrual to the main randomization was completed on January 15, 2016. As of March 14, 2016, the projected relapse event horizon was reached and outcome analyses indicated that the addition of bortezomib did not improve EFS, DFS or OS, but did demonstrate a higher incidence of 
non-fatal treatment-related toxicities. Therefore, institutions were notified on this date that patients receiving protocol therapy on the bortezomib arm should switch to the standard chemotherapy arm immediately. There were 22 patients who were receiving protocol therapy on the bortezomib arm at this time.
Table 1 and Online Supplementary Table S1 summarizes the demographic characteristics of patients by study arm; no significant differences were observed in these demgraphic characteristics. Of note, $33 \%$ and $13 \%$ of patients had favorable cytogenetic or molecular features, respectively, and $<5 \%$ had unfavorable cytogenetic features.

Table 1. Patient demographics and clinical characteristics by treatment arm.

\begin{tabular}{|c|c|c|c|c|c|c|c|}
\hline \multirow[t]{2}{*}{ Characteristic } & \multicolumn{2}{|c|}{ Overall } & \multicolumn{2}{|c|}{ Arm A } & \multicolumn{2}{|c|}{ Arm B } & \multirow[t]{2}{*}{$P$} \\
\hline & N & $\%$ & N & $\%$ & N & $\%$ & \\
\hline \multicolumn{8}{|l|}{ Gender } \\
\hline Male & 572 & $52 \%$ & 285 & $53 \%$ & 287 & $52 \%$ & 0.773 \\
\hline Female & 525 & $48 \%$ & 257 & $47 \%$ & 268 & $48 \%$ & \\
\hline \multicolumn{8}{|l|}{ Age at diagnosis, years } \\
\hline Median & 9.2 & & 9.5 & & 9.1 & & 0.511 \\
\hline Range & $0-29.5$ & & $0.03-29.5$ & & $0-29.2$ & & \\
\hline 0-1 [0-730 day old] & 237 & $22 \%$ & 107 & $20 \%$ & 130 & $23 \%$ & 0.139 \\
\hline $2-10$ & 372 & $34 \%$ & 189 & $35 \%$ & 183 & $33 \%$ & 0.507 \\
\hline $11-15$ & 273 & $25 \%$ & 139 & $26 \%$ & 134 & $24 \%$ & 0.565 \\
\hline $16-20$ & 188 & $17 \%$ & 91 & $17 \%$ & 97 & $17 \%$ & 0.763 \\
\hline$\geq 21$ & 27 & $2 \%$ & 16 & $3 \%$ & 11 & $2 \%$ & 0.300 \\
\hline \multicolumn{8}{|l|}{ Race } \\
\hline American Indian or Alaskan Native & 9 & $1 \%$ & 3 & $1 \%$ & 6 & $1 \%$ & 0.506 \\
\hline Asian & 51 & $5 \%$ & 24 & $5 \%$ & 27 & $6 \%$ & 0.699 \\
\hline Native Hawaiian or other Pacific Islander & 8 & $1 \%$ & 3 & $1 \%$ & 5 & $1 \%$ & 0.726 \\
\hline Black or African American & 136 & $14 \%$ & 69 & $14 \%$ & 67 & $14 \%$ & 0.793 \\
\hline White & 767 & $79 \%$ & 384 & $80 \%$ & 383 & $78 \%$ & 0.652 \\
\hline Multiple Races & 1 & $0 \%$ & 0 & $0 \%$ & 1 & $0 \%$ & 1.000 \\
\hline Unknown & 125 & & 59 & & 66 & & \\
\hline \multicolumn{8}{|l|}{ Ethnicity } \\
\hline Hispanic or Latino & 199 & $19 \%$ & 99 & $19 \%$ & 100 & $19 \%$ & 0.945 \\
\hline Not Hispanic or Latino & 863 & $81 \%$ & 427 & $81 \%$ & 436 & $81 \%$ & \\
\hline Unknown & 35 & & 16 & & 19 & & \\
\hline \multicolumn{8}{|l|}{ 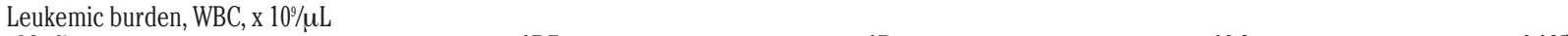 } \\
\hline Median & 17.7 & & 17 & & 19.2 & & 0.185 \\
\hline Range & $0.6-2730$ & & $0.6-2730$ & & $0.6-2600$ & & \\
\hline N. of patients with $>100 \times 10^{9} / \mu \mathrm{L}$ & & $16 \%$ & & $16 \%$ & 93 & $17 \%$ & 0.620 \\
\hline \multicolumn{8}{|l|}{ CNS disease classification at study entry } \\
\hline CNS1 & 730 & $69 \%$ & 358 & $70 \%$ & 372 & $69 \%$ & 0.617 \\
\hline CNS2 & 215 & $20 \%$ & 100 & $20 \%$ & 115 & $21 \%$ & 0.507 \\
\hline CNS3 & 108 & $10 \%$ & 53 & $10 \%$ & 55 & $10 \%$ & 0.905 \\
\hline Unknown & 44 & & 31 & & 13 & & \\
\hline Non-CNS extramedullary disease & 170 & $15 \%$ & 82 & $15 \%$ & 88 & $16 \%$ & 0.720 \\
\hline \multicolumn{8}{|l|}{ Risk factors and classification } \\
\hline \multicolumn{8}{|l|}{ Cytogenetics affecting risk classification } \\
\hline $\mathrm{t}(8 ; 21)$ & 166 & $20 \%$ & 84 & $16 \%$ & 82 & $15 \%$ & 0.725 \\
\hline $\operatorname{Inv}(16), t(16 ; 16)$ & 114 & $13 \%$ & 57 & $11 \%$ & 57 & $11 \%$ & 0.883 \\
\hline-7 & 21 & $3 \%$ & 9 & $2 \%$ & 12 & $2 \%$ & 0.545 \\
\hline$-5 / 5 q-$ & 13 & $1 \%$ & 6 & $1 \%$ & 7 & $1 \%$ & 0.814 \\
\hline \multicolumn{8}{|l|}{ Institution mutation results } \\
\hline Low FLT3-ITD allelic ratio $(\leq 0.4)$ & 77 & $7 \%$ & 37 & $7 \%$ & 40 & $7 \%$ & 0.805 \\
\hline NPM & 80 & $7 \%$ & 37 & $7 \%$ & 43 & $8 \%$ & 0.558 \\
\hline $\mathrm{CEBP} \alpha$ & 66 & $6 \%$ & 29 & $5 \%$ & 37 & $7 \%$ & 0.364 \\
\hline \multicolumn{8}{|l|}{ MRD at end of induction I } \\
\hline Negative & 782 & $75 \%$ & 386 & $75 \%$ & 396 & $75 \%$ & 0.929 \\
\hline Positive & 261 & $25 \%$ & 128 & $25 \%$ & 133 & $25 \%$ & \\
\hline MRD positive \%, median & 2.3 & & 2.8 & & 1.9 & & 0.247 \\
\hline MRD positive \%, range & $0.1-93$ & & $0.1-93$ & & $0.1-92$ & & \\
\hline Unknown & 54 & & 28 & & 26 & & \\
\hline \multicolumn{8}{|l|}{ Risk group assignment } \\
\hline Low & 836 & $78 \%$ & 417 & $79 \%$ & 419 & $78 \%$ & 0.664 \\
\hline High & 230 & $22 \%$ & 111 & $21 \%$ & 119 & $22 \%$ & \\
\hline
\end{tabular}

AML: acute myeloid leukemia; CNS: central nervous system; ITD high AR: internal tandem duplication with high allelic ratio; MRD: minimum residual disease;WBC: white blood cell count. 
Minimal residual disease (MRD) assessment at the end of Induction I was available in $95 \%$ of patients, and was negative in $75 \%$. Thus, approximately $78 \%$ of all patients were classified as low-risk based on cytogenetic, molecular, and disease response features, while $22 \%$ were classified as high-risk.

Of the 1,097 patients enrolled on AAML1031, approximately $84 \%$ survived and achieved a remission at the end of two courses of induction. For the 1,024 patients who initiated the second course of induction therapy and were evaluable at the end of Induction II, the remission rate was $90 \%$ and there was no difference between study arms. No differences in EFS and OS were observed by study arm (Table 2 and Figure 2). Specifically, the 3-year EFS from study entry for the no bortezomib and bortezomib arms were $44.8 \% \pm 4.5 \%$ versus $47.0 \% \pm 4.5 \%(P=0.236)$ and the

Table 2. Event-free survival, overall survival, and treatment-related mortality by study arm.

\begin{tabular}{lccccccc} 
& \multicolumn{2}{c}{ Overall } & \multicolumn{2}{c}{ Arm A } & \multicolumn{3}{c}{ Arm B } \\
& N & $\% \pm 2$ SE\% & N & $\% \pm 2$ SE\% & N & \% \pm 2 SE\% \\
3-year EFS from study entry & 1097 & $45.9 \pm 3.2$ & 542 & $44.8 \pm 4.5$ & 555 & $47.0 \pm 4.5$ & 0.236 \\
3-year OS from study entry & 1097 & $65.4 \pm 3.1$ & 542 & $63.6 \pm 4.5$ & 555 & $67.2 \pm 4.3$ & 0.356 \\
\hline 3-year CI of relapse from study entry & 1097 & $47.2 \pm 3.2$ & 542 & $48.0 \pm 4.5$ & 555 & $46.4 \pm 4.4$ & 0.378 \\
1-year TRM from study entry & 1097 & $11.8 \pm 5.2$ & 542 & $13.3 \pm 8.2$ & 555 & $10.5 \pm 6.6$ & 0.577 \\
\hline 3-year DFS from end of Induction I & 1015 & $47.8 \pm 3.3$ & 506 & $46.9 \pm 4.6$ & 509 & $48.7 \pm 4.6$ & 0.261 \\
3-year OS from end of Induction I & 1015 & $66.6 \pm 3.2$ & 506 & $65.2 \pm 4.6$ & 509 & $68.0 \pm 4.5$ & 0.451 \\
3-year DFS from end of Induction II & 910 & $52.4 \pm 3.5$ & 453 & $51.8 \pm 4.9$ & 457 & $53.0 \pm 4.9$ & 0.444 \\
3-year OS from end of Induction II & 910 & $70.5 \pm 3.3$ & 453 & $69.3 \pm 4.8$ & 457 & $71.7 \pm 4.7$ & 0.453 \\
1-year TRM from end of Induction II & 910 & $9.7 \pm 5.2$ & 453 & $10.4 \pm 7.6$ & 457 & $9.0 \pm 7.2$ & 0.331 \\
\hline
\end{tabular}

EFS: event-free survival; OS: overall survival; CI: cumulative incidence;TRM: treatment-related mortality; DFS: disease-free survival; SE: standard error.

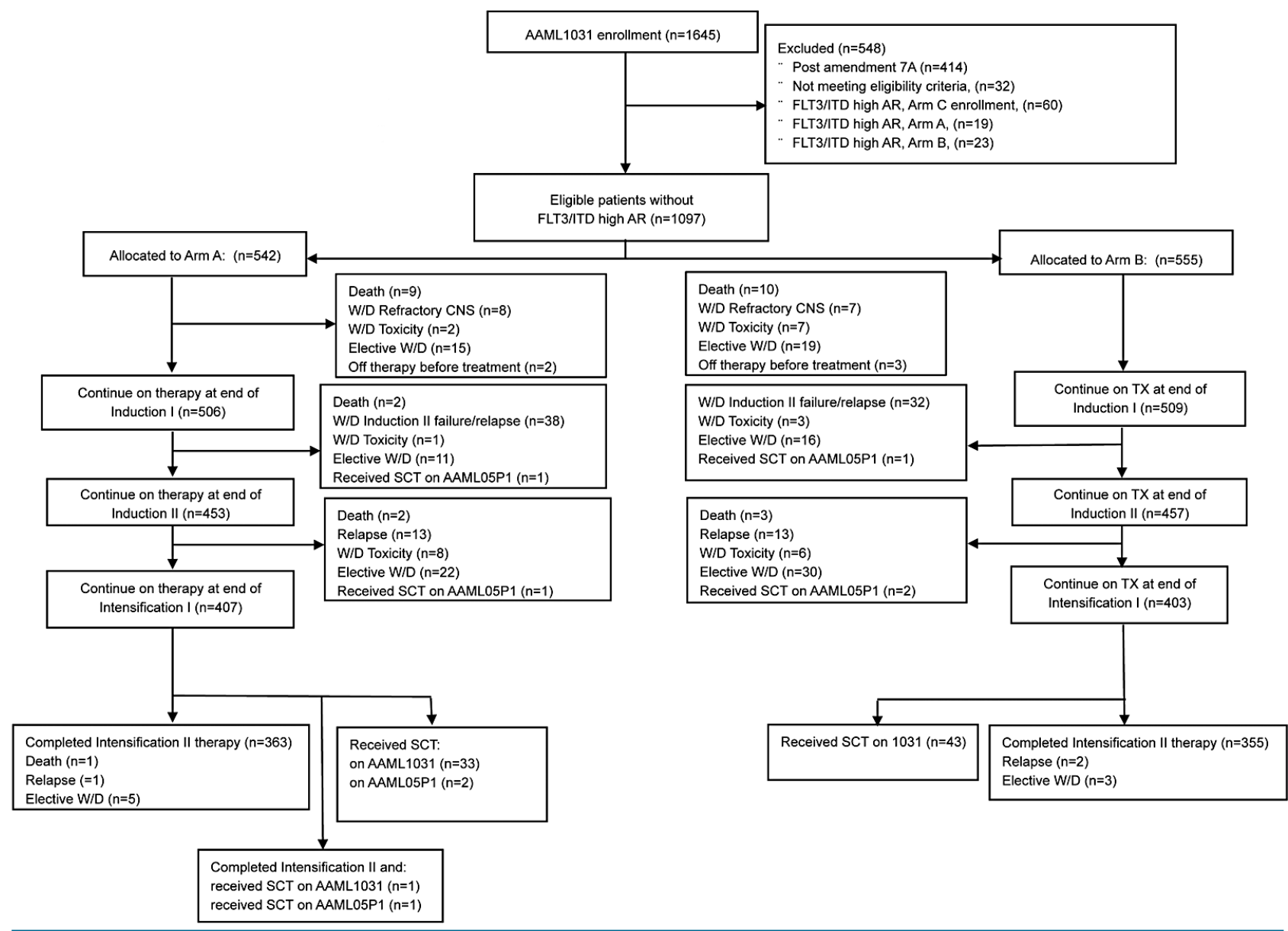

Figure 1. Consort diagram - AAML1031, as of December 31, 2017. High AR: W/D: Elective withdrawal. Reasons include terminating therapy to due to physician's choice or patient's refusal of further protocol therapy. SCT: stem cell transplantation; TX: therapy; n: number. 
3-year OS from study entry were $63.6 \% \pm 4.5 \%$ versus $67.2 \% \pm 4.3 \%(P=0.356)$. Similar outcomes by randomization arm were observed for the cumulative incidence of relapse, 1-year TRM, and DFS/OS from the end of Induction II (Table 2).

Subgroup analyses by risk group (Online Supplementary Table S2) showed similar outcomes between treatment arms for both low- and high-risk patients. Combining the two arms, 3-year DFS and OS for low-risk patients was $52.9 \% \pm 3.7 \%$ and $74.1 \% \pm 3.4 \%$, respectively while 3 -year DFS and OS for high-risk patients was $27.8 \% \pm 6.6 \%$ and $36.9 \% \pm 7.6 \%$. Subgroup analyses by NPM, CEBPA, CBF, and KMT2A molecular subtypes (Online Supplementary Table S3) and by age category (Online Supplementary Table S4) did not show any evidence of subtype- or age-specific bortezomib responses.

Univariable and multivariable Cox analyses from study entry and end of Induction II are shown in Table 3 and Online Supplementary Table S5. Initial white blood cell count $(\mathrm{WBC})>100 \times 10^{9} / \mathrm{L}$ was significantly associated with an increased risk of relapse, treatment-related mortality, and decreased survival from study entry. Age greater or equal to 11 years old was associated with a decreased risk of relapse and increased survival. Black race, a previously observed risk factor, ${ }^{3,19}$ was no longer a significant risk factor for relapse or death. The magnitude and significance of these associations remained stable between univariate and multivariable analyses.

Interim analyses of TRM and acute respiratory distress syndrome (ARDS) after 100 patients were randomized to bortezomib did not cross predefined toxicity thresholds. Overall TRM and targeted toxicity data are shown in Table 4 and Online Supplementary Table S6. No differences were observed in overall or course-specific TRM. While most toxicity rates did not differ by treatment arm, peripheral neuropathy, dose reductions, and pediatric Intensive Care Unit (PICU) admissions were consistently increased in patients receiving bortezomib in combination with standard chemotherapy. Course-specific increased rates of ARDS and hypoxia were observed in the patients treated with bortezomib together with standard chemotherapy. However, the reported rates of these toxi- cities was relatively low and did not differ from rates in patients treated with standard chemotherapy alone. No differences in infectious complications, renal toxicities, or decline in shortening fraction/ejection fraction were observed between treatment arms (Online Supplementary Table S7). Subgroup toxicity analyses by patient age demonstrated increased toxicities in Arm B patients with increasing age (Online Supplementary Table S8) amongst patients who completed all four courses of chemotherapy.

\section{Discussion}

The AAML1031 trial data demonstrate that the addition of bortezomib to standard chemotherapy does not improve EFS or OS. However, bortezomib caused additional treatment-related toxicity, specifically peripheral neuropathy, dose reductions, and PICU admissions. Given the lack of clinical benefit and increased toxicity observed in the bortezomib treatment arm, bortezomib was discontinued in all patients who remained on protocol mandated therapy. While the preliminary data regarding bortezomib efficacy in adults with AML was promising, ${ }^{14-16}$ and pediatric preclinical models demonstrated a potential biological rationale for combining bortezomib with pediatric AML chemotherapy, ${ }^{12,13}$ the results of AAML1031 do not support the addition of bortezomib to current pediatric AML chemotherapy. This trial result illustrates the need for specific pediatric clinical trials in AML, even in the context of a promising efficacy signal in adult AML.

Several important additional conclusions may be drawn from these data. First, the outcomes seen on the AAML1031 trial are generally similar to those seen on the standard arm of the immediately antecedent phase III trial, AAML0531, and are slightly inferior to outcomes reported in other pediatric co-operative oncology groups. ${ }^{3,20-22}$ The observed differences in outcomes between other pediatric co-operative oncology group clinical trials and AAML1031 are still not completely understood but stem, in part, from the elimination of chemotherapy cycle 5 (Capizzi AraC) for low-risk patients with uninformative molecular features. ${ }^{23}$ Further investigations will evaluate differences in

Table 3. Multivariable analyses.

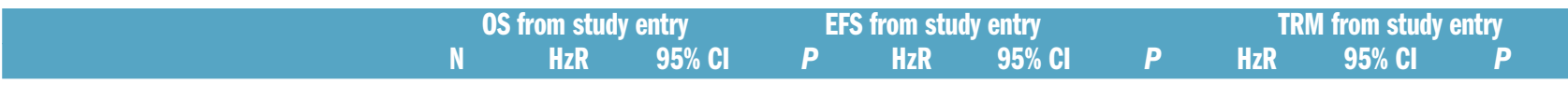

Treatment arm

Arm A

Arm B

$482 \quad 1$

Age at diagnosis, years

2-10

0-1

487

0.91

$0.73-1.13$

0.383

1

1

$\begin{array}{lllllllllll}0-1 & 209 & 1.26 & 0.94-1.68 & 0.118 & 1.21 & 0.96-1.53 & 0.100 & 0.80 & 0.32-1.99 & 0.638 \\ \geq 11 & 442 & 0.86 & 0.66-1.11 & 0.231 & 0.78 & 0.64-0.96 & 0.017 & 1.25 & 0.65-2.40 & 0.498\end{array}$

WBC at diagnosis, $\mathrm{x} 10^{\circ} / \mathrm{L}$

\begin{tabular}{|c|c|c|c|c|c|c|c|c|c|c|}
\hline$\leq 100$ & 805 & 1 & & & 1 & & & 1 & & \\
\hline$>100$ & 164 & 1.42 & $1.08-1.86$ & 0.013 & 1.64 & $1.32-2.03$ & $<0.001$ & 1.79 & $0.92-3.48$ & 0.089 \\
\hline \multicolumn{11}{|l|}{ Race } \\
\hline Non-black & 832 & 1 & & & 1 & & & 1 & & \\
\hline Black & 137 & 1.30 & $0.97-1.75$ & 0.084 & 1.02 & $0.79-1.31$ & 0.884 & 1.86 & $0.95-3.62$ & 0.068 \\
\hline
\end{tabular}

OS: overall survival; EFS: event-free survival;TRM: treatment-related mortality; HzR: hazard ratio; CI: confidence interval;WBC: white blood cell count. 
Table 4. Targeted toxicity by phase of therapy.

\begin{tabular}{|c|c|c|c|c|c|c|c|c|c|c|c|c|c|}
\hline & Phase of therapy & & nduction & & & iduction & & & ensifica & tion I & & insificat & \\
\hline & Treatment arm & Arm A & Arm B & $\begin{array}{c}\text { A vs. B } \\
P\end{array}$ & Arm A & & $\begin{array}{c}\text { A vs. B } \\
P\end{array}$ & Arm A & Arm B & $\begin{array}{c}\text { A vs. B } \\
P\end{array}$ & Arm A & Arm B & $\begin{array}{c}\text { A vs. B } \\
P\end{array}$ \\
\hline Toxicity & $\mathrm{N}$ & 574 & 580 & & 518 & 529 & & 460 & 469 & & 373 & 361 & \\
\hline Cardiac & Heart failure & 4 & 4 & & 3 & 6 & & 5 & 10 & & 10 & 13 & \\
\hline & & $0.7 \%$ & $0.7 \%$ & 1.000 & $0.6 \%$ & $1.1 \%$ & 0.506 & $1.1 \%$ & $2.1 \%$ & 0.206 & $2.7 \%$ & $3.6 \%$ & 0.474 \\
\hline & EF decreased & 4 & 6 & & 1 & 5 & & 8 & 19 & & 4 & 11 & \\
\hline & & $0.7 \%$ & $1.0 \%$ & 0.753 & $0.2 \%$ & $0.9 \%$ & 0.218 & $1.7 \%$ & $4.1 \%$ & 0.036 & $1.1 \%$ & $3.0 \%$ & 0.059 \\
\hline & Cardiac LVSD & 5 & 8 & & 4 & 4 & & 13 & 15 & & 9 & 16 & \\
\hline & & $0.9 \%$ & $1.4 \%$ & 0.413 & $0.8 \%$ & $0.8 \%$ & 1.000 & $2.8 \%$ & $3.2 \%$ & 0.740 & $2.4 \%$ & $4.4 \%$ & 0.132 \\
\hline Neurologic & Peripheral neuropathy/ & 6 & 20 & & 4 & 17 & & 8 & 14 & & 5 & 10 & \\
\hline & Paresthesia/neuralgia & $1.0 \%$ & $3.4 \%$ & 0.006 & $0.8 \%$ & $3.2 \%$ & 0.005 & $1.7 \%$ & $3.0 \%$ & 0.212 & $1.3 \%$ & $2.8 \%$ & 0.171 \\
\hline & Seizure & 2 & 1 & & 1 & 0 & & 0 & 0 & & 0 & 3 & \\
\hline & & $0.3 \%$ & $0.2 \%$ & 0.623 & $0.2 \%$ & $0.0 \%$ & 0.495 & $0.0 \%$ & $0.0 \%$ & 1.000 & $0.0 \%$ & $0.8 \%$ & 0.119 \\
\hline Pulmonary & ARDS & 2 & 12 & & 2 & 3 & & 6 & 3 & & 3 & 1 & \\
\hline & & $0.3 \%$ & $2.1 \%$ & 0.008 & $0.4 \%$ & $0.6 \%$ & 1.000 & $1.3 \%$ & $0.6 \%$ & 0.337 & $0.8 \%$ & $0.3 \%$ & 0.624 \\
\hline & Нyрохіа & 21 & 35 & & 7 & 10 & & 7 & 24 & & 15 & 17 & \\
\hline & & $3.7 \%$ & $6.0 \%$ & 0.060 & $1.4 \%$ & $1.9 \%$ & 0.490 & $1.5 \%$ & $5.1 \%$ & 0.002 & $4.0 \%$ & $4.7 \%$ & 0.648 \\
\hline & Respiratory failure & 10 & 18 & & 2 & 3 & & 4 & 5 & & 8 & 5 & \\
\hline & & $1.7 \%$ & $3.1 \%$ & 0.133 & $0.4 \%$ & $0.6 \%$ & 1.000 & $0.9 \%$ & $1.1 \%$ & 1.000 & $2.1 \%$ & $1.4 \%$ & 0.435 \\
\hline Renal & Acute kidney injury & 9 & 10 & & 0 & 4 & & 1 & 6 & & 2 & 1 & \\
\hline & & $1.6 \%$ & $1.7 \%$ & 0.835 & $0.0 \%$ & $0.8 \%$ & 0.124 & $0.2 \%$ & $1.3 \%$ & 0.124 & $0.5 \%$ & $0.3 \%$ & 1.000 \\
\hline & Creatinine increased & 0 & 5 & & 1 & 2 & & 0 & 2 & & 0 & 1 & \\
\hline & & $0.0 \%$ & $0.9 \%$ & 0.062 & $0.2 \%$ & $0.4 \%$ & 1.000 & $0.0 \%$ & $0.4 \%$ & 0.500 & $0.0 \%$ & $0.3 \%$ & 0.492 \\
\hline Microbiologically & Viridans group & 21 & 25 & & 55 & 53 & & 70 & 78 & & 83 & 75 & \\
\hline $\begin{array}{l}\text { documented } \\
\text { sterile site infections } \\
\text { (at least } 1 \text { occurrence) }\end{array}$ & Streptococcus & $3.7 \%$ & $4.3 \%$ & 0.572 & $10.6 \%$ & $10.0 \%$ & 0.750 & $15.2 \%$ & $16.6 \%$ & 0.556 & $22.3 \%$ & $20.8 \%$ & 0.627 \\
\hline & Gram Negative Bacilli & 9 & 16 & & 23 & 31 & & 41 & 49 & & 53 & 43 & \\
\hline & & $1.6 \%$ & $2.8 \%$ & 0.165 & $4.4 \%$ & $5.9 \%$ & 0.299 & $8.9 \%$ & $10.4 \%$ & 0.429 & $14.2 \%$ & $11.9 \%$ & 0.356 \\
\hline & Fungi & 16 & 7 & & 3 & 7 & & 0 & 2 & & 6 & 6 & \\
\hline & & $2.8 \%$ & $1.2 \%$ & 0.055 & $0.6 \%$ & $1.3 \%$ & 0.342 & $0.0 \%$ & $0.4 \%$ & 0.500 & $1.6 \%$ & $1.7 \%$ & 0.955 \\
\hline Dose reductions & & 8 & 31 & & 8 & 33 & & 4 & 37 & & 9 & 47 & \\
\hline & & $1.4 \%$ & $5.3 \%$ & $<0.001$ & $1.5 \%$ & $6.2 \%$ & $<0.001$ & $0.9 \%$ & $7.9 \%$ & $<0.001$ & $2.4 \%$ & $13.0 \%$ & $<0.001$ \\
\hline PICU admissions & & $\begin{array}{c}121 \\
21.1 \%\end{array}$ & $\begin{array}{c}155 \\
26.7 \%\end{array}$ & 0.025 & $\begin{array}{c}43 \\
8.3 \%\end{array}$ & $\begin{array}{c}66 \\
12.5 \%\end{array}$ & 0.027 & $\begin{array}{c}53 \\
11.5 \%\end{array}$ & $\begin{array}{c}84 \\
17.9 \%\end{array}$ & 0.006 & $\begin{array}{c}72 \\
19.3 \%\end{array}$ & $\begin{array}{c}71 \\
19.7 \%\end{array}$ & 0.901 \\
\hline
\end{tabular}

ARDS: adult respiratory distress syndrome; EF: ejection fraction; LVSD: left ventricular cystolic dysfunction; PICU: pediatric intensive care unit.

study populations, including characteristics such as obesity, molecularly-defined risk differences between study populations, efficacy of the backbone treatment regimen, variations in supportive care practices, and the potential impact of structural differences in the provision of health services. Additional analyses including comparisons focusing on the efficacy of dexrazoxane as a cardioprotectant ${ }^{24}$ specific cytogenetic abnormalities (MLL translocation subgroups), the use of $\mathrm{MRD}$ testing for outcome prediction, optimizing risk classification, the intensification of Induction II therapy with cytarabine and mitoxantrone, and the role of allogeneic donor SCT, are ongoing.

Second, COG, in partnership with the Cancer Therapy Evaluation Program (CTEP) can conduct complex clinical trials that contain phase I, phase II, and phase III components. The sorafenib study arm, which will be reported separately, served as a phase I trial of the feasibility and initial efficacy assessment of incorporating sorafenib into pediatric AML. Moreover, at the initiation of AAML1031, the only published data for bortezomib in pediatric AML was as a single agent. ${ }^{17}$ While limited safety data were available during the 2-year planning process prior to the opening of the AAML1031 trial in June, 2011, full safety and efficacy data were not available until the subsequent closure of the AAML07P1 trial in December, 2011. ${ }^{18}$ Given these limited toxicity data, the AAML1031 trial included a planned targeted toxicity (ARDS and TRM) analyses after the randomization of 100 patients to the bortezomib treatment arm. The successful monitoring of bortezomib-associated toxicities on the AAML1031 trial highlights the ability of COG, in partnership with the CTEP, to conduct complex clinical trials that provide definitive efficacy testing of a novel agent in the setting of limited preliminary toxicity data. 


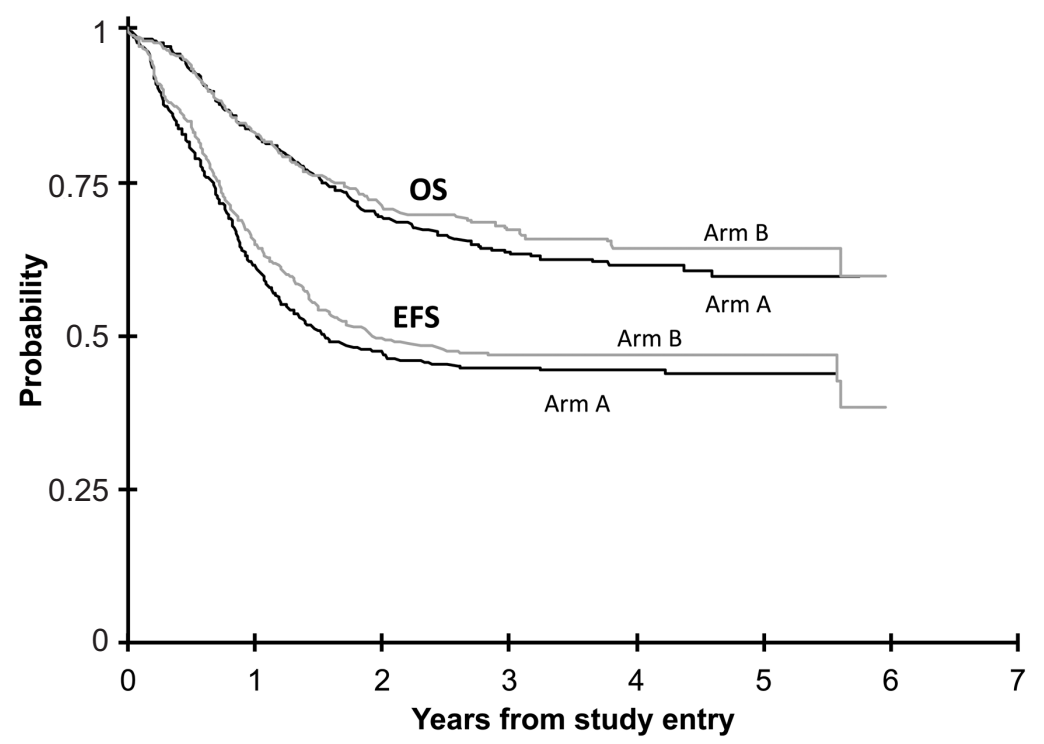

Figure 2. Event-free survival (EFS) and overall survival (OS) by treatment arm.

Several limitations of this clinical trial require acknowledgment. First, correlative biology data on the unfolded protein response and other biomarkers of bortezomib efficacy are currently ongoing and thus could not be included in this report. These ongoing studies may define subgroup populations who may benefit from bortezomib. ${ }^{25}$ Second, comprehensive molecular profiling of each individual AML case is ongoing but is still not complete. ${ }^{26}$ The completion of this work will likely enable the next generation of risk prediction and therapy individualization. Finally, the ongoing analyses of changes in chemotherapy course sequence and use of allogeneic donor SCT will face the well documented challenges of limitations in chemotherapy toxicity reporting, ${ }^{27,28}$ and the challenges faced by all cooperative oncology groups to collect and account for variable supportive care practices and particular factors at the level of each individual center that may impact treatment outcomes.

In conclusion, the AAML1031 trial demonstrates that bortezomib can be combined safely with standard pediatric AML chemotherapy but that this combination does not improve EFS or OS and is associated with increased toxicity. Thus, these data do not support the use of bortezomib in pediatric AML therapy at this time. Despite this, the successful conduct of this very complex trial highlights the clinical trial capabilities of COG in partnership with the CTEP, and may serve as a paradigm for definitive efficacy clinical trials initiated in the setting of limited preliminary data. Finally, the AAML1031 clinical trial data set, in conjunction with ongoing biology studies, will serve as an invaluable data platform for future clinical and translational investigations.

\section{Acknowledgments}

The AAML1031 study team would like to acknowledge the patients and families who participated in the AAML1031 clinical trial.

\section{Funding}

This research was supported by NCTN Operations Center Grant (U10CA180886) and NCTN Statistics \& Data Center Grant (U10CA180899).

\section{References}

1. Zwaan CM, Kolb EA, Reinhardt D, et al. Collaborative Efforts Driving Progress in Pediatric Acute Myeloid Leukemia. J Clin Oncol. 2015;33(27):2949-2962.

2. Tarlock K, Meshinchi S. Pediatric acute myeloid leukemia: biology and therapeutic implications of genomic variants. Pediatr Clin North Am. 2015;62(1):75-93.

3. Gamis AS, Alonzo TA, Meshinchi S, et al. Gemtuzumab ozogamicin in children and adolescents with de novo acute myeloid leukemia improves event-free survival by reducing relapse risk: results from the randomized phase III Children's Oncology Group trial AAML0531. J Clin Oncol. 2014; 32(27):3021-3032.

4. Cooper TM, Franklin J, Gerbing RB, et al. AAML03P1, a pilot study of the safety of gemtuzumab ozogamicin in combination with chemotherapy for newly diagnosed childhood acute myeloid leukemia: A report from the children's oncology group. Cancer. 2011;118(3):761-769.

5. Lange BJ, Smith FO, Feusner J, et al. Outcomes in CCG-2961, a children's oncology group phase 3 trial for untreated pediatric acute myeloid leukemia: a report from the children's oncology group. Blood. 2008;111(3):1044-1053

6. Gamis AS, Alonzo TA, Perentesis JP, Meshinchi S, Committee COGAML. Children's Oncology Group's 2013 blueprint for research: acute myeloid leukemia. Pediat Blood Cancer. 2013;60(6):964-971.

7. Matondo M, Bousquet-Dubouch MP, Gallay N, et al. Proteasome inhibitorinduced apoptosis in acute myeloid leukemia: a correlation with the proteasome status. Leuk Res. 2010;34(4):498506.

8. Colado E, Alvarez-Fernandez S, Maiso P, et al. The effect of the proteasome inhibitor bortezomib on acute myeloid leukemia cells and drug resistance associated with the CD34+ immature phenotype. Haematologica. 2008;93(1):57-66.

9. Guzman ML, Neering SJ, Upchurch D, et al. Nuclear factor-kappaB is constitutively activated in primitive human acute myelogenous leukemia cells. Blood. 2001; 98(8):2301-2307.

10. Guzman ML, Swiderski CF, Howard DS, et al. Preferential induction of apoptosis for primary human leukemic stem cells. Proc Natl Acad Sci U S A. 2002;99(25):1622016225.

11. Jordan CT, Guzman ML, Noble M. Cancer stem cells. N Engl J Med. 2006;355(12): 1253-1261.

12. Horton TM, Gannavarapu A, Blaney SM, et al. Bortezomib interactions with chemotherapy agents in acute leukemia in vitro. Cancer Chemother Pharmacol. 2006; 
58(1):13-23.

13. Niewerth D, Franke NE, Jansen G, et al. Higher ratio immune versus constitutive proteasome level as novel indicator of sensitivity of pediatric acute leukemia cells to proteasome inhibitors. Haematologica. 2013;98(12):1896-1904.

14. Attar EC, De Angelo DJ, Supko JG, et al. Phase I and pharmacokinetic study of bortezomib in combination with idarubicin and cytarabine in patients with acute myelogenous leukemia. Clin Cancer Res. 2008; 14(5):1446-1454.

15. Blum W, Schwind S, Tarighat SS, et al. Clinical and pharmacodynamic activity of bortezomib and decitabine in acute myeloid leukemia. Blood. 2012;119(25): 6025-6031.

16. Orlowski RZ, Voorhees PM, Garcia RA, et al. Phase 1 trial of the proteasome inhibitor bortezomib and pegylated liposomal doxorubicin in patients with advanced hematologic malignancies. Blood. 2005; 105(8):3058-3065.

17. Horton TM, Pati D, Plon SE, et al. A phase 1 study of the proteasome inhibitor bortezomib in pediatric patients with refractory leukemia: a Children's Oncology Group study. Clin Cancer Res. 2007;13(5):15161522.

18. Horton TM, Perentesis JP, Gamis AS, et al. A Phase 2 study of bortezomib combined with either idarubicin/cytarabine or cytarabine/etoposide in children with relapsed refractory or secondary acute myeloid leukemia: a report from the Children's Oncology Group. Pediatric Blood Cancer. 2014;61(10):1754-1760

19. Aplenc R, Alonzo TA, Gerbing RB, et al Ethnicity and survival in childhood acute myeloid leukemia: a report from the Children's Oncology Group. Blood. 2006; 108(1):74-80.

20. Hasle H, Abrahamsson J, Forestier E, et al. Gemtuzumab ozogamicin as postconsolidation therapy does not prevent relapse in children with AML: results from NOPHOAML 2004. Blood. 2012;120(5):978-984.

21. Creutzig U, Zimmermann M, Bourquin JP, et al. Randomized trial comparing liposomal daunorubicin with idarubicin as induction for pediatric acute myeloid leukemia: results from Study AML-BFM 2004. Blood. 2013;122(1):37-43

22. Tsukimoto I, Tawa A, Horibe K, et al. Riskstratified therapy and the intensive use of cytarabine improves the outcome in childhood acute myeloid leukemia: the AML99 trial from the Japanese Childhood AML Cooperative Study Group. I Clin Oncol. 2009;27(24):4007-4013

23. Getz KD, Alonzo TA, Sung L, et al. Four versus five chemotherapy courses in patients with low risk acute myeloid leukemia: a Children's Oncology Group report. J Clin Oncol. 2017; 35(15_suppl): 10515-10515.

24. Getz KD, Sung L, Leger K, et al. Effect of dexrazoxane on left ventricular function and treatment outcomes in patients with acute myeloid leukemia: A Children's Oncology Group report. J Clin Oncol. 2018; 36(15_suppl):10501-10501.

25. Hoff FW, Oiu Y, Hu W, et al. Abstract 451 Proteomic profiling of the unfolded protein response identifies patients benefiting from bortezomib in pediatric acute myeloid leukemia. Cancer Res. 2018;78(Suppl 13):451.

26. Bolouri $\mathrm{H}$, Farrar JE, Triche $\mathrm{T} J \mathrm{r}$, et al. The molecular landscape of pediatric acute myeloid leukemia reveals recurrent structural alterations and age-specific mutational interactions. Nat Med. 2018;24(1):103112.

27. Miller TP, Li Y, Getz KD, et al. Using electronic medical record data to report laboratory adverse events. Br J Haematol. 2017 177(2):283-286.

28. Miller TP, Troxel AB, Li Y, et al. Comparison of administrative/billing data to expected protocol-mandated chemotherapy exposure in children with acute myeloid leukemia: A report from the Children's Oncology Group. Pediatric Blood Cancer. 2015;62(7):1184-1189. 